\title{
Ocrelizumab
}

\section{Therapie der Multiplen Sklerose}

Die FDA verlieh dem Medikament Ocrelizumab den Status Therapiedurchbruch.

Quelle:

Presseaussendung

Roche Austria GmbH
Dem Prüfmedikament Ocrelizumab für die Behandlung von Patienten mit primär progredienter Multipler Sklerose (PPMS) hat die US-amerikanische Zulassungsbehörde den Status Therapiedurchbruch verliehen. Damit ist Ocrelizumab das erste Mittel gegen PPMS, das diesen Status erhält.

Der Status des Therapiedurchbruchs soll die Entwicklung und Prüfung von Medikamenten für schwerwiegende oder lebensbedrohliche Erkrankungen beschleunigen und sicherstellen, dass Patienten durch die FDA-Zulassung so schnell wie mög- lich Zugang zu diesen Medikamenten erhalten. Die Voraussetzung im Falle von Ocrelizumab waren positive Resultate der zulassungsrelevanten Phase-III-Studie ORATORIO. Darin wurde gezeigt, dass die Behandlung mit Ocrelizumab das Fortschreiten der Behinderung und andere Marker der Krankheitsaktivität, verglichen mit einem Placebo, signifikant reduzierte. Ocrelizumab ist ein humanisierter monoklonaler Antikörper, der selektiv und gezielt gegen CD20-positive B-Zellen gerichtet ist, die vermutlich wesentlich zur Schädigung der Myelinscheide und der Axone beitragen. Wie präklinische Studien gezeigt haben, bindet Ocrelizumab an CD20-Oberflächenproteine, die auf bestimmten B-Zellen exprimiert werden, nicht jedoch auf Stammzellen oder Plasmazellen. Deshalb bleiben wichtige Funktionen des Immunsystems erhalten.

Roche plant die Marktzulassung sowohl für PPMS als auch für schubförmige MS und wird in der ersten Jahreshälfte 2016 die Daten von drei wichtigen Phase-III-Studien bei den Zulassungsbehörden weltweit einreichen.

\section{Kindliche Demenz}

\section{Erstmals medikamentöse Therapie}

Enzymersatztherapie zur Behandlung einer speziellen Form von kindlicher Demenz.

CLN2 gilt als unheilbar. Die Krankheit gehört zur Gruppe der neuronalen Ceroid-Lipofuszinosen $(\mathrm{NCL})$, einer frühen und rasch fortschreitenden Form von Kinder-Demenz, bei der die Patienten ab dem 3. bis 5. Lebensjahr rapide ihre Fähigkeit zu laufen und zu sprechen verlieren und früh versterben. Nun liegen Studienergebnisse einer medikamentösen Enzymersatztherapie zur Behandlung vor: Studienleiterin Dr. Angela Schulz aus dem Universitätsklinikum Hamburg-Eppendorf (UKE) erklärte, dass die unheilbare Krankheit CLN2 bei $87 \%$ der 24 behandelten Kinder deutlich langsamer voranschritt als bei unbehandelten Patienten. Erstmalig konnte eine fortschreitend verlaufende Form von Kinder-Demenz erfolgreich behandelt werden.

Von September 2013 bis Dezem- der- und Jugendmedizin des UKE eine sogenannte klinische Phase 1/2-Studie zur intraventrikulären Enzymersatz-Therapie bei CLN2-Krankheit durchgeführt. Im Rahmen der Studie wurde den Patienten ein künstlich hergestelltes Enzym in zweiwöchigem Abstand über 48 Wochen hinweg mit Hilfe eines kleinen Katheterschlauchs in den Hirninnenraum verabreicht, um so das fehlende Enzym zu ersetzen und das Absterben der Nervenzellen zu stoppen. Dieses Vorgehen wurde weltweit erstmals im Rahmen dieser Studie durchgeführt. Zwölf von insgesamt 24 Studienpatienten weltweit wurden in Hamburg behandelt, weitere Studienzentren waren in Rom, London, und Columbus, USA.

Die Sicherheit und Verträglichkeit der Behandlung wurde bei allen 24 Patienten als positiv bewertet. Darüber hinaus konnte ein signifikanter Be- handlungserfolg gezeigt werden: $65 \%$ der Patienten erreichten über 48 Wochen eine Stabilisierung der Fähigkeit zu laufen und zu sprechen und $87 \%$ der Patienten zeigten ein deutlich langsameres Fortschreiten der Krankheit verglichen mit Daten unbehandelter Patienten. Kernspin-Untersuchungen des Gehirns zeigten, dass behandelte Kinder signifikant weniger des Volumens ihrer Hirnrinde pro Jahr verlieren als unbehandelte Kinder. Die Studie habe den Beweis erbracht, dass eine Enzymersatztherapie, die direkt in den Hirninnenraum verabreicht wird, wirksam sein kann, so Schulz. Dies stellt einen lang ersehnten Hoffnungsschimmer für CLN2-Patienten weltweit dar.

psychopraxis. neuropraxis 2016 · 19:66 DOI 10.1007/s00739-016-0317-y Online publiziert: 15. März 2016 (c) Springer-Verlag Wien 2016 